\title{
6 Beantwortung der Fragestellungen
}

In dieser Arbeit wurde gezeigt, dass das reflektierende Gespräch die Kompetenzentwicklung unterstützen kann (Frage 1). Dabei ist es einerseits sinnvoll, bereits im Studium die Studierenden in der Reflexion über Denk- und Entscheidungsprozesse in der therapeutischen Arbeit zu unterstützen und gleichzeitig an die Evidenzbasierte Praxis im Sinne der Handlungsorientierung anzuknüpfen, weil diese beiden Elemente für die logopädische Tätigkeit entscheidend sind. Neben den Basiskompetenzen sollten also berufsbezogene Kompetenzen wie die Anwendung des Clinical Reasonings und die Arbeit in Anlehnung an die Evidenzbasierte Praxis bereits im Studium fokussiert werden (Frage 3). Durch wertschätzende Gesprächsführungstechniken und lösungsorientierte Fragen kann das Kompetenzentwicklungsgespräch positiv beeinflusst werden, damit Studierende ihre Ressourcen aktivieren und Perspektiven herausarbeiten können (Frage 2). Durch den Einsatz eines Reflexionsbogens mit vorbereitenden Fragen können Ängste und Unsicherheiten auf der Seite der Studierenden verringert werden. Zudem wird der Fokus auf die Reflexion der eigenen Kompetenzentwicklung gelenkt (Frage 4). Die kombinierte Selbst- und Fremdbeobachtung zählt zu den qualitativen Kompetenzmessverfahren. Da das Ziel die Einschätzung der Lernbewegung der Studierenden ist, die ihre Handlungen reflektieren und ggf. neu ausrichten sollen, macht diese Innenschau vollkommen Sinn, vor allem dann, wenn der Blick auf sich selbst durch die Außensicht des Lehrlogopäden ergänzt wird, was einen Abgleich ermöglicht. In dieser Arbeit wurde auf die Skalierbarkeit der Fragen im Sinne eines hybriden Verfahrens verzichtet, weil nicht der Bogen analysiert werden sollte, sondern der Ermöglichungsrahmen des reflektierenden Gesprächs. Damit ist die Kompetenzentwicklung nicht objektiv nachweisbar. Es wird aber angenommen, dass in Form von mündlichen und schriftlichen Prüfungen weiterhin Kompetenzentwicklung gemessen wird, so dass die subjektive Beurteilung der Studierenden als Ausnahme von der Regel sogar wünschenswert erscheint und eine weitere Perspektive darstellt (Frage 5). Ergänzend könnten die Studierenden am Ende des Gesprächs dazu befragt werden, ob sich durch die Vorbereitung auf das Gespräch und das Gespräch selbst die Einschätzung der eigenen Kompetenzen verändert hat. Diese Frage könnte man empirisch auswerten, was aber über diese Arbeit hinausgeht. 